\title{
Understanding Mental Health: What Are the Issues for Black and Ethnic Minority Students at University?
}

\author{
Jason Arday \\ School of Education, University of Roehampton, London SW15 5PU, UK; Jason.arday@roehampton.ac.uk
}

Received: 12 August 2018; Accepted: 8 October 2018; Published: 13 October 2018

\begin{abstract}
Black and Minority Ethnic (BME) communities continue to experience inequalities within the United Kingdom (UK) mental health system despite major government policy initiatives. Access to higher education for many ethnic minorities remains problematic. Within higher education, BME students consistently face barriers in terms of accessing culturally appropriate services including a lack of cultural understanding, communication issues, and where and how to seek help. This paper attempts to address the problems facing ethnic minorities with regard to accessing mental health services at university. Importantly, this paper highlights that barriers to accessing mental health support for ethnic minorities directly impact upon attainment outcomes and psychological well-being. This study utilizes the narratives of 32 BME university students to examine the impact of negotiating racial inequality and discrimination at university and the impact upon mental health. Aspects examined considered the impact of belonging, isolation, and marginalization on mental health and how this consequently affects university participation for BME students. Utilizing a thematic analysis paradigm, the key findings presented point towards differential healthcare outcomes for ethnic minority university students experiencing mental illness. The empirical findings in this paper suggest that currently ethnic minority service users experience overt discrimination and a lack of access to culturally appropriate services that are cognizant of the racialized plights faced by BME individuals. These findings inform an overarching dialogue, which suggests that mental health service providers need to work more collegially with people from BME communities prior to service design and delivery. Furthermore, the findings suggest that, upon presenting mental health issues, information should be made available in appropriate languages for ethnic minorities to support understanding about their illnesses and how they can seek professional intervention and help. Conclusions and recommendations provided advocate greater diversification of mental health support systems for ethnic minority students within universities. Conclusions drawn will also consider how existing systems can function to dismantle racial inequality within the mental health profession.
\end{abstract}

Keywords: black and ethnic minority (BME); ethnic minorities; service user; healthcare provider; racism; diversification; inequality

\section{Introduction}

Increasing diagnoses of mental illness globally have thrust the importance of mental health into public consciousness. The emphasis on mental health has gained widespread attention as healthcare professionals attempt to find more effective ways of managing mental illness (MIND 2013). Ethnic inequalities in mental health continue to be highlighted as a cause for concern within numerous policy documents and reviews published or commissioned by the United Kingdom (UK) Government. 
Research (Grey et al. 2013; Wallace et al. 2016) shows that many Black and Minority Ethnic (BME) ${ }^{1}$ groups experience significant variation when accessing mental health care pathways in the UK. This is reflected in some BME groups being less likely to be referred to mental health services through their General Practitioner (GP) and more likely to be arrested by the police following a crisis, which inevitably results in poorer health outcomes and often-coercive forms of care in locked wards. Within higher education, there has become a narrative of crisis regarding an ethnic minority student well-being and mental health, which has seen an increasing impact upon degree attainment and retention (Equality Challenge Unit 2015; Tate and Bagguley 2017).

The importance of mental health should occupy a position of prominence within the everyday language of higher education discourse since this permeates every aspect of the student experience. For ethnic minorities' navigating the racialized terrain of the Academy, there is a dearth of culturally sensitive interventions within the UK that allow BME students to openly discuss their experiences of mental health without fear of further discrimination, reprisal, or judgement (Arday 2017). Consequently, this has impacted confidence among ethnic minority students in being able to present potential well-being issues and access support within universities and the wider health care system (MIND 2013). The prevalence of common mental disorders varies markedly in different BME communities. For example, within a university context, South Asian women encounter higher rates of anxiety and depression (63.5\% compared with $28.5 \%$ of white women) and among Afro-Caribbean men $3.1 \%$ compared with $0.2 \%$ of white men encounter psychotic disorders or episodes but are less likely to well-served by mental health support services particularly when feelings of isolation and marginalization begin to emerge (MIND 2013). Within universities, there has been an emerging pattern regarding the utilization of mental health services among ethnic minority groups, which points towards differential and inequitable experiences and outcomes. In general, people from ethnic minorities are less likely than their white British counterparts to have contacted their General Practitioner (GP) about mental health issues resulting in a greater likelihood of being prescribed anti-depressants or being referred to specialist mental health services.

People from minority ethnic communities have historically been underrepresented in health research (Giuliano et al. 2000). The impact of racialization historically has been impactful on ethnic minorities attempting to navigate a discriminatory landscape permeated by institutionally racist structures (Andrews 2016). Within ethnic minority communities, there are cultural differences regarding the way mental health is perceived, accepted, and acknowledged. Globally, there is a stigmatization that has accompanied mental illness. However, raising awareness of this context has often been situated within a dominant white and Eurocentric backdrop (Grey et al. 2013; Sewell 2012). Addressing this imbalance to ensure that people from all sections of society are represented equally is important in reflecting mental illness as a problem that affects all types of people regardless of race, class, religion, gender, sexuality, or disability (Glover and Evison 2009; Vernon 2011). There are two significant factors that contribute to how mental illness is framed societally.

First, there is a failure within existing mental health research to ensure that all sections of society are equally represented. Research in this area repeatedly fails to acknowledge the salient differences in how ethnic minority groups experience mental health in comparison to the entire United Kingdom (UK) population (Rugkåsa and Canvin 2011). Such factors include psychological issues that are commonly

1 Commentators suggest the use of precise descriptions regarding the ethnic background when describing research findings (Bradby 2003; McKenzie and Crowcroft 1996). For the purposes of this paper, the term Black and Minority Ethnic and the abbreviation BME will be used to refer to people who are from ethnic backgrounds other than white British (including black African, African Caribbean, Asian, Latin-American, and other minority ethnic communities) with more precise descriptions used where appropriate. There is a recognition, however, that the term BME is not universally accepted. Although, this is the term commonly used within the British vernacular. It is important to acknowledge that the term BME, despite its widespread use, has severe limitations and usually follows non-specific quantifiers such as 'most' or 'some' (Glover and Evison 2009). Typically, there has been an accepted use of the term BME, which has been illustrated in research and Government papers. Given the purpose of this paper, this term is applied purely as a descriptive term having been the preferred term for most of the participants throughout this study. 
found in particular racial groups that could be a consequence of continuously encountering varying forms of discrimination (Wallace et al. 2016). Invariably, potential differences in the experiences of such issues within and between population sub-groups place an emphasis on the continual development of effective services or interventions for minority groups (Yancey et al. 2006). Secondly, there is a social justice element that encompasses the dialogue of mental illness, which has often omitted ethnic minorities from mental health research (Yancey et al. 2006). The imbalance in representation indirectly perpetuates existing power imbalances and inequities, which does not ensure that ethnic minorities receive the same types of cognitive treatment and rehabilitation, which sometimes results in patients being incorrectly diagnosed and heavily medicated (Benoit et al. 2005; Cooper et al. 2013).

In its most rudimentary form, mental health is the culmination of everything that comes into human awareness (Norman and Ryrie 2009). Norman and Ryrie (2009) assert that the safeguarding and promotion of good mental health is paramount for all communities within British society. Developing mental health interventions that are beneficial for ethnic minorities is essential particularly when we consider the racial ascriptions and objectification of black people and black men in particular within Britain and global society more generally (Grey et al. 2013; Memon et al. 2016). The impact of this often affects access to opportunity most notably in education (attainment outcomes) and employment. In the UK, black men are over-represented at the coercive end of the mental health system but under-represented in terms of seeking help voluntarily (Myrie and Gannon 2013). Statutory and non-statutory organizations within the UK have often highlighted the need to address inequalities in a mental health service provision for ethnic minorities (Care Quality Commission 2011; Department of Health 2010). Presently, there are similarities in the discriminatory experiences of Black and Minority Ethnic (BME) groups within the mental health system and higher education. BME services users have expressed dissatisfaction with mental health services given the fact that they are more likely to be subject to over-diagnoses, which often results in admissions to medium-security and high-security psychiatric wards/clinics with less emphasis placed on offering psychologically-based interventions (Jones and Berry 1986; Myrie and Gannon 2013). Similarly, within higher education, there are significant rates of dissatisfaction particularly in relation to student experience, belonging, curriculum, and the BME degree attainment gap deficit (Arday and Mirza 2018; Equality Challenge Unit 2015). BME students often report experiences of exclusion in varying forms particularly concerning student experiences that are not culturally accepting, sensitive, or diverse. The impact upon belonging is significant since ethnic minority students will inevitably withdraw themselves from these exclusionary spaces (Alexander and Arday 2015; Arday 2017). The marginalization of ethnic minority students within the university space has historically been exacerbated by the proliferation of dominant Eurocentric curricula presented as the central body of knowledge that students must ascertain (Andrews 2016; Arday and Mirza 2018). Many of these factors ultimately affect the learning experience of ethnic minority students who encounter adverse experiences in terms of assessment, access to help, supervision. and support consequently impacting upon degree attainment (Pilkington 2013). In considering these aspects, there is an under-appreciation within the academy that BME students encounter and experience university differently and, consequently, holistic and academic needs will differ. The lack of acknowledgment within the sector does pose a significant risk to the mental and physical well-being of ethnic minority students attempting to navigate themselves through university.

As with most forms of discrimination and inequality for ethnic minorities, racism often occupies a position of centrality in their marginalized and oppressed experiences. There is evidence that attitudes towards mental health concerning BME people are often conflated within cultural contexts that advocate 'resilience' and 'strength' over presenting and confronting mental health concerns (Grey et al. 2013). Stigmatization within these communities is problematic and this is exacerbated when we consider attitudes towards psychological distress and the accessibility of resources available to ethnic minority populations (Memon et al. 2016). Traversing this discriminatory terrain can be a 
precursor to BME communities being more likely to struggle and less likely to receive the psychological support required.

Mental health and educational attainment within higher education particularly for BME learners have become interwoven against a backdrop of institutional racism. In many cases, BME students will have already traversed a somewhat problematic education system that has historically failed ethnic minority communities and, in particular, young, black boys (Andrews 2016). The terrain traversed can be traumatic and can cause mental fatigue, which can often reveal itself in the form of lower attainment, increased anxieties, and cultural pressures particularly for young, ethnic minority women (Mirza 2017). The landscape of higher education within the United Kingdom (UK) presents a very complex and somewhat contradictory discourse. Higher education within the UK is often represented as a vehicle for inclusion, diversity, and multi-culturalism (Alexander 2017). However, the contradictory position that emerges often ignores the racial discrimination that resides within the academy, which disadvantages BME students in addition to the structures that impact aspects of attainment and engagement (Bhopal 2014). Against a backdrop of societal and sometimes cultural ideologies that tend to present mental illness as showing fear, distress, or displays of emotionality, attitudes towards mental illness concerning BME individuals can be difficult (Grey et al. 2013). For BME students undertaking university study and negotiating mental illness, many of them will already be managing overwhelming family pressure and expectation to attain good degrees in the hope that this will potentially pave the way for a successful professional career (Arday 2017). This paper examines the impact of negotiating racial inequality and discrimination at a university and the impact upon mental health. Aspects examined consider the impact of belonging, isolation, and marginalization on mental health and how this consequently affects university participation for BME students. Unpacking the stigma of mental illness among BME communities requires an understanding of some of the cultural pressures faced by ethnic minority students upon entering and transitioning through university. Illuminating some of these factors also provides opportunities to examine the service user and healthcare provider experiences while at university.

For ethnic minorities, social factors play a significant role in facilitating patterns of racialized oppression. Isolation and marginalization are widely acknowledged to be linked with the onset of mental health problems (Vernon 2011). The oppression faced can often be a precursor to mental illness and this becomes compounded for many individuals from BME backgrounds required to be continuously resilient in the face of enduring institutional racism. Importantly, this paper will argue that, against a backdrop of continuous inequality and discrimination, BME students endure severe consequences in attempting to successfully negotiate university. As such, it is unsurprising that ethnic minorities are at an increased risk of mental health issues (Grey et al. 2013). Cultural alienation from wider communities within universities and society become undoubtedly a significant contributing factor that gradually affects and de-stabilizes mental and psychological well-being amongst ethnic minorities (Ahmed 2012).

Lastly, this paper concludes by providing suggestions and recommendations for how mental health support systems can be better diversified for ethnic minority people within universities. Conclusions drawn will also consider how existing systems can function to dismantle racism. Concluding thoughts will proffer that ethnic minorities encounter continuous discrimination and institutional racism throughout varying societal structures, which leaves ethnic minorities more vulnerable to mental illness in the first place.

\section{The Problem}

The prevalence of student mental health issues has gleaned significant attention with students encountering ever-increasing pressures associated within good degree attainment, debt, and gaining employment. Sadly and rather worryingly, the current plight has coincided with an increase in suicide attempts by university students. The extent of BME and white differences regarding student mental health is significant. Research undertaken by the mental health charity MIND (2013) suggests 
that nearly two-thirds of ethnic minority students experiencing mental illness often experience discriminatory encounters with healthcare professionals. Furthermore, MIND (2013) states that ethnic minority students already encounter hyper-surveillance within societal spaces, which heightens feelings of sensibility and vulnerability. The prejudiced scrutiny of environmental and socialization factors has often resulted in diagnoses that advocate medication above cognitive therapies and coping strategies for ethnic minorities (MIND 2013).

Comparatively, white students are often presented with a variety of cognitive and behavioral therapies aimed at not developing a dependency on medication instead of focusing on promoting continuity and stability in managing mental illness. White university students do continue to experience mental health support differently with MIND (2013) finding that white students often report positive experiences with mental health professionals that are situated around several self-help interventions and building confidence within patients to create autonomous individuals that become aware of the potential triggers that may affect their mental health and physical well-being.

An aspect of higher education that often underpins the BME student experience are feelings of isolation and marginalization (Arday 2017; Rollock 2016). The way in which this isolation is experienced creates a hyper-consciousness, which can be exacerbated by unwanted surveillance often resulting in a solitary experience for BME students in predominantly white environments within the university space (Bhopal 2014). In many cases, Ahmed (2012) suggests that this isolation can be become intensified when discussing culturally sensitive information, which can impact aspects of well-being negatively. Representation creates a sense of belonging and, for many BME students, the paucity of diversification is often reflected within staff and student populations most notably at the Russell Group ${ }^{2}$ institutions, which remain overwhelmingly white with regard to staff and student concentration (Alexander 2017; Alexander and Arday 2015). The lack of diversification has wider implications for the mental health of BME students who may feel reluctant to disclose their issues considering the dearth in representation and relatability racially and culturally (Equality Challenge Unit 2015; Shilliam 2015). Significantly, these factors will inevitably impact upon attainment particularly when considering the interactions that students will often need to have with academic tutors to ensure their successful transition through university. Tate and Bagguley (2017) explain that many BME students find it difficult to express race-related issues to academics because there tends to be a cultural and contextual disconnect in attempting to understand the nuances of racialized experiences.

\subsection{Why Does This Matter?}

The plight of BME mental health within higher education and wider society points to a failing of institutional services that have a designated remittance to support the mental well-being of these individuals. Within health-related research, people from ethnic minority communities continue to be under-represented. As a direct consequence, there remains a dearth of literature that has examined extensively the effects of mental health on BME communities within professional environments. Recent offerings (Memon et al. 2016; MIND 2013; Vernon 2011) have attempted to highlight the consequences of mental health issues and inequalities when not adequately addressed regarding equitable experiences within higher education. Wallace et al. (2016) suggest that inequitable access to mental health services for ethnic minority students create a discerning chasm between receiving support and becoming progressively more entrenched within the perils of mental illness. Furthermore, Wallace et al. (2016) assert that ethnic minority students often do not receive the culturally sensitive help and support required from health care professionals and, as a consequence, will become

2 'The Russell Group is a self-selected association of 24 public research universities in the United Kingdom. The group is headquartered in London and was established in 1994 to represent its members' interests principally to Government and Parliament. Representing the UK's leading universities, the Russell Group has historically been committed to maintaining its status as research intensive in addition to having unrivaled links with business and the public sector (The Russell Group 2018). 
reliant upon themselves to negotiate mental illness. This is problematic because the immediate intervention and diagnosis of mental illness is essential in arresting the early phases of symptoms associated with depression, anxiety, and psychosis. The equitable experience in higher education is further compromised for ethnic minority students who have not been given access to the type of non-judgmental mental health provision provided to white students (Fernando 2010). The lack of equitable access for BME students submerged within the debilitating stranglehold of mental illness will enviably impact attainment outcomes and equality of experience in terms of personal and professional development while navigating university life (Alexander and Arday 2015; Memon et al. 2016).

The absence of extensive networks for ethnic minority groups is a pertinent factor in the participation of such mental health research (Davies et al. 2009, Shavers et al. 2001). Attempting to glean those voices that have experienced mental illness during university becomes problematic when we consider the cycle of marginalization and isolation that many BME students face upon entering university as self-preservation strategies move from attainment to survival (Picower 2009). For many university students, the stigma of mental illness often means that individuals are reluctant to disclose any issues they may have (Wilkinson and Pickett 2010). The backdrop to this particular reoccurring narrative has tended to be situated within building trust in pastoral interventions provided by universities, which do not pre-judge or label ethnic minorities before cognitive behavioral processes have been utilized (Vernon 2011).

The lack of trust in research or in the medical system is often mentioned by BME communities that have experienced these particular services (Giuliano et al. 2000). Shavers et al. (2001) found that often BME individuals are less willing to participate in medical research in comparison to their white counterparts. There are other practical issues that affect the participation of BME communities within this type of research. Skepticism from BME communities can impact this context significantly particularly for BME university students who may be carrying the burden of cultural and communal expectations while attempting to assimilate into university life (Yancey et al. 2006). Other commentaries have espoused reasons for the exclusion of BME participants from mental health surveys and interventions. Williams (2005) suggested that some research institutions have established recruitment networks that do not include BME groups. Others (Grey et al. 2013, Memon et al. 2016; Myrie and Gannon 2013; Williams 2005) have frequently reported that additional barriers include language needs and the lack of recognition that healthcare services require modernizing to facilitate the diverse needs of an ever-increasing multi-cultural society. In considering structures or barriers to healthcare services, this inadvertently becomes another way of marginalizing BME communities who are in need of potential psychological or mental health support (Vernon 2011).

Unpacking this dialogue when situated within a higher education context is challenging particularly when we consider the ways in which higher education attempts to exclude ethnic minorities (Bhopal 2014; Andrews 2016). For BME students within universities, the centrality of whiteness is continuously facilitated through aspects of curricula, poor diversification, and racial ascriptions regarding the attainment capabilities of ethnic minority students (Alexander and Arday 2015; Law 2017). Navigating the inequitable terrain for BME students can become mentally exhausting and taxing, which subsequently impacts aspects of attainment (Equality Challenge Unit 2015). Furthermore, this becomes problematic because attitudes towards mental illness within BME communities can be restrictive with discourses often situated in developing greater resilience or establishing a greater connection with faith (religious) as the only reliable and viable intervention in arresting mental health decline (Alvarez et al. 2006; Keating and Robertson 2004). Keating and Robertson (2004) explains that culturally, we exist within a society that has historically been guilty of trivializing mental illness as mental frailty rather than as a legitimate illness that destabilizes individuals' mental facilities for short or prolonged periods of time. For BME students, these trivializations of mental illness make it increasingly difficult to disclose some of the crippling psychological symptoms of mental illness. Aspects of loneliness, marginalization, and isolation can become accelerated as ethnic minorities attempt to deal with this issue on their own, which ultimately plunges them 
into further difficulties without the correct coping mechanisms (Keating 2007). Within a higher education context, ever-increasing student populations have also placed a strain on pastoral services and resources at universities, as they attempt to cater for increasing student populations. Illuminating the ethnic minority voice is particularly important in challenging how we change dominant norms, which often omit how BME individuals experience mental health within society and throughout their journeys within education (Grey et al. 2013; Rugkåsa and Canvin 2011). Gleaning this voice provides the stimulus for how we revaluate discussions regarding mental health among ethnic minorities. Wendler et al. (2006) detected very small differences in willingness to take part between ethnic groups in their review of published research that reported consent rates (in mainly quantitative surveys) by race or ethnicity. They concluded that, rather than focusing on changing the attitudes of minority groups, it is necessary to promote access to health research for all groups. However, it is safe to assert that the catalyst to changing this narrative is dictated by how we better engage ethnic minorities in disclosing experiences of mental illness (MIND 2013). Initially, Fernando (2003) suggests that the primary focus should involve providing more inclusive and accessible platforms for ethnic minorities to enable disclosures regarding mental illness. However, as alluded to earlier, this is normally dependent on specific cultural contexts or traditions. Ahmed (2012) explains that relationship building is essential in creating spaces of belonging and disclosure for BME communities that have historically felt on the periphery of some of society's major institutions. There is a consensus that advocates the building of relationships through community representatives regarding BME voices and their mental health (Fernando 2010). The traction gathered regarding greater calls for better representation and diversification within universities in terms of academic representation and student service representation becomes integral particularly in terms of building synergy and familiarity culturally for ethnic minority students (Rollock 2016; Shilliam 2015).

\subsection{The Causes}

The heightened and relatively recent pressures attributed to navigating university within the UK have significantly impacted ethnic minority students who, in many cases, will have already traversed systemic and institutional racism in some form throughout their lives (Alexander and Arday 2015). Precipitating factors such as racial ascription, stereotyping, stigmatization, discrimination, hyper-surveillance, and a lack of access to opportunities regarding employment exacerbate mental health issues for ethnic minorities. For BME students, these factors will invariably impact their worldview and, as a consequence, mental wellness can become disrupted or compromised. The academy, as already discerned, is an exclusive rather than inclusive space that has often marginalized BME individuals through various instruments of discrimination such as curricula, unconscious bias, and poor cultural diversification (Andrews 2016). Wallace et al. (2016) explain that, for BME students attempting to navigate university life, these factors become cumulative and the dearth of specific and non-judgmental mental support interventions deepens issues concerning access to equitable mental health provisions for ethnic minorities within universities. Continuously encountering these discriminatory factors has contributed to the increase of mental health diagnoses among ethnic minority students (Braun and Clarke 2006; Memon et al. 2016).

While some BME students will arrive at universities with mental health difficulties, there has tended to be paucity of pastoral interventions with an ethnic focus, which have meant that ethnic minorities in particular have struggled to gain the required support needed to navigate or manage their mental illness (Grey et al. 2013). Furthermore, Arday and Mirza (2018) suggest that the inequitable nature of universities for ethnic minorities can cause mental health difficulties or exacerbate them.

Enrichment activities traditionally at universities in the form of clubs and societies can often ostracize BME students especially those that are non-inclusive of other cultures. Often, there can be potential cultural clashes which emerge regarding university enrichment activities (facilitated via university Student Unions) that may conflict with cultural or religious beliefs. This leaves many BME students feeling marginalized as they struggle to assimilate into these somewhat exclusionary spaces 
(Ahmed 2012; Pilkington 2013). Vernon (2011) emphasizes that such racialized experiences heighten feelings of angst, which is a tenet of mental illness. As a minority voice with no outlet to express these feelings, the university experience becomes fraught, which consequently affects attainment as many BME students will endure systemic forms of racism. This may be overt or covert among some of their peers and academic staff (Equality Challenge Unit 2015; Shilliam 2015).

Student accommodation has also proven to be a place where BME students face experiences of discrimination and exclusion, which can often result in ethnic minorities spending large periods of time on their own without avenues to converse with others who may be experiencing similar feelings of ostracism (Andrews 2016; Shilliam 2015). Vernon (2011) explains that, in new environments, belonging becomes essential in the initial integration process with regards to meeting new people where some individuals feel they are on the periphery of these environments. This can create feelings of angst, marginalization, and exclusion. Fundamentally, the emphasis on universities as micro-communities requires re-thinking particularly when considering how certain minority groups remain on the periphery of these spaces. LGBTQ groups, religious groups, individuals with disability, and ethnic minorities are some examples of these minority groups. It is important to note that cross-cultural acquaintances can differ for men and women. A study undertaken by the University of Bristol with BME students at the university indicated that $66 \%$ of black men found it easier to assimilate themselves within the university through integration with clubs and societies. Conversely, this differed for black women with $71 \%$ stating that they found it difficult to assimilate themselves within the university through integration with clubs and societies.

In constructing the narrative of marginalization and exclusion and the implications for mental health and attainment, it is important to acknowledge the impact of dominant Eurocentric curricula, which often omits ethnic minorities (Andrews 2016; Mirza 2017). Curricula becomes a significant catalyst for ethnic minorities developing a sense of belonging within universities particularly if that curriculum reflects their worldview or life experience historically and presently. Unpacking diverse perspectives for many BME students can be cathartic in relieving mental health pressures particularly when trying to navigate the university space (Grey et al. 2013).

\subsection{The Barriers}

The barriers faced by ethnic minority students at university often make it difficult for mental health difficulties to be confronted and addressed during the initial presentation of potential issues. The lack of access for BME students to mental health services reported (Equality Challenge Unit 2015; MIND 2013) often suggests that fear of further stigmatization or racial discrimination is a significant reason as to why ethnic minorities find it harder to access help and support within university spaces. Alexander and Arday (2015) explain that BME students will already be aware that they occupy a vulnerable and isolated position within a predominantly dominant white environment. Therefore, access to pastoral services will be difficult to access and retrieve against this particular backdrop. Andrews (2016) suggests that the diversification of pastoral services within universities would positively impact the BME student experience particularly if the support was culturally-sensitive and targeted.

The well-being of BME students within universities is paramount for their attainment and general integration within these spaces as a minority. Lent (2004) situates well-being as an essential but often neglected area within psychological health when considering the initiation of ethnic minority students within universities. Furthermore, Fernando (2010) states that, within university spaces, a wide variety of factors make it difficult to distinguish the crucial elements of well-being particularly with regards to negating the complex needs for varying minority groups. Understanding and safeguarding this well-being remains the responsibility of universities particularly in the case of BME students who continue to experience occurrences of exclusion that impact them upon successful integration into university life (Alexander 2017; Law 2017). Addressing these factors for universities is imperative because, in the United Kingdom (UK), there are higher rates of mental health problems in BME communities compared with the national average (MIND 2012). The dominant discourse, 
which precedes this context, has often situated BME communities with mental health problems to be more likely than others to receive a diagnosis of severe mental illness, to experience involuntary treatment, and commonly to enter mental health services via the criminal justice system (Keating 2007; McKenzie et al. 2001; MIND 2013). The dialogue of severe mental health diagnosis is often conflated within this stereotypical context, which can mean that ethnic minorities can be subject to inaccurate and negative summative psychological assessments. This makes many individuals from BME communities reluctant to disclose any potential mental illnesses for fear of stigmatization against this dominant discourse (Keating and Robertson 2004). Unfortunately, such debates tend to be the precursor for some of the racially ascribed views held concerning the ethnic minority experience with mental illness. Fernando (2010) states that there needs to be a wider recognition that acknowledges that, when discussing issues concerning mental health with BME communities, this particular context has historically been underpinned with differential factors concerning 'socio-economic hardship' and 'power' between 'minority' and 'majority' ethnic communities. Importantly, while the issue of stigma surrounding mental health problems and disclosure might sound like a familiar one, discourses concerning mental health and psychological wellness as a taboo subject rarely consider cultural and communal factors that make disclosure far more difficult for ethnic minority students particularly within universities (Palmer and Ward 2007; Wilkinson and Pickett 2010).

Unpacking this within the context of higher education, particularly when considering diversity, attention can often gravitate towards representation at the level of admissions, curricula, and lecturers, but it is easy to overlook that crucially our support structures and services-that is, our nurses, our officers, our tutors, our centers, and our counsellors-form the basis for successful student progression (Shilliam 2015). Within higher education, a more renewed and penetrative focus is required to ensure that these existing services provide better outcomes for ethnic minority students (Equality Challenge Unit 2015).

\subsection{The Solutions}

Potential solutions require universities to advocate greater diversification within pastoral and mental healthcare provision. Memon et al. (2016) emphasize that there is a cultural cognizance and sensitivity that is required when ethnic minority students present symptoms associated with mental health difficulties. The historic absence of this awareness has been a significant contributing factor in the difficultly experienced by ethnic minorities in utilizing these services (Wallace et al. 2016). Universities must develop interventions that advocate more culturally inclusive spaces since the paucity of these spaces exacerbate feelings of marginalization and isolation for ethnic minorities who are potentially already feeling to be on the periphery of an exclusionary university. Interventions must seek to understand how ethnic minorities experience mental illness differently from white individuals and the impact of cultural pressure and expectation on this phenomena (Fernando 2010; Vernon 2011).

Re-imagining and rethinking our 'safe spaces' within universities is a reoccurring theme that has gained traction throughout the sector as considerations shift towards how greater diversification within the sector can be achieved (Alexander and Arday 2015; Bhopal 2014; Equality Challenge Unit 2015). Importantly, Ahmed (2012) explains that this ideology needs to be better situated within an inclusive dialogue that is able to facilitate varying multi-ethnic groups. Safe spaces, by definition, are non-judgmental, confidential spaces where people can disclose personal thoughts, feelings, and issues that might be affecting their well-being (MIND 2013). Therapy similarly functions as a safe and confidential space. However, Keating (2007) explains that ethnic minorities are rarely well-represented within therapeutic settings, wider support structures, or psychological services and, thus, working through experiences of mental illness becomes harder. Equally 'safe spaces' for BME individuals can become another discriminatory instrument to being further misunderstood and marginalized (Wilkinson and Pickett 2010). The onus on universities to improve mental health provisions regarding ethnic minorities is paramount at this particular juncture as the indifferent treatment of BME students within the sector gains more traction regarding attainment outcomes, 
integration, decolonizing the curriculum, greater diversification, and access to pastoral services and provision (Arday 2017).

\section{Materials and Methods}

For the purpose of this study, 14 UK based-universities were used ranging from the Russell Group to Post- $92^{3}$ institutions. BME individuals $(n=32)$ aged between 18-34 years old were recruited from undergraduate and postgraduate degree programs. Thirty-two semi-structured, open-ended questionnaires were completed initially to capture participants' ethnic origin, gender, and age range. This was solely for monitoring purposes. The questionnaire also assisted in gleaning general information concerning experiences with mental health either personally or with friends or family to help inform the development of the focus group and individual interview questions. In addition, we included 2 unstructured focus group interviews and 32 40-min semi-structured individual interviews involving all 32 participants to explore lived experiences of negotiating mental illness as BME students within higher education. The recruitment of participants was facilitated through recommendations with several African and Caribbean university societies with social media platforms used to enlist ethnic minority participants. Additionally, convenience sampling was used to diversify the pool of participants to ensure that the sample was as representative as possible regarding the ethnic minority demographic to be considered (Cohen et al. 2011).

The initial part of the study involved each participant being given an anonymous self-administered questionnaire to complete, which was deposited into a ballot box. The questionnaire included information on the following: gender, age (in age groups), level of education (up until degree level), ethnicity (according to the Office of National Statistics classification), marital status, and duration of time within higher education. The results reported in this paper draw primarily on excerpts from the two focus group discussions (each lasting for about $3 \mathrm{~h}$ ), which included 32 participants from the following ethnicities: Asian/Asian British $(n=6)$, black/black British $(n=14)$, mixed-heritage $(n=9)$, and Latin-American $(n=3)$. The study was comprised of 18 females and 14 males. The overwhelming majority of participants attended a university where the majority of students were white.

The focus groups were conducted in April 2017 within the UK. The objectives of the study were explained to the participants and informed consent was obtained. Discussions were facilitated by the researcher who had experience in cross-cultural working and qualitative methods. All focus group sessions and interviews were audio-recorded and transcribed verbatim. In addition to the recorded discussions, written notes were also taken and flipcharts were utilized for participants to document patterns of thought. This facilitated a reflexive process, which ensured participants' views were clearly documented. Each participant was encouraged to speak and express their own views. The supportive and nurturing environment cultivated candid conversations among participants, which enriched the disclosure process.

A topic/discussion guide with questions developed and led by the researcher was used to ascertain various aspects of access to mental health services at a university and externally within respective local communities including type of service(s) used, issues with, and experience of using the service, perceived barriers to accessing the service, and how healthcare services can be improved for ethnic minorities within university spaces where often BME students tend to feel marginalized or isolated. Broad topic guide questions included: (1) What are your perspectives on mental health? (2) How do ethnic minorities deal with mental health issues? (3) Do you think mental health and psychological services are made accessible to BME individuals within universities and wider society more generally? (4) Culturally, how might encounters with mental illness differ for ethnic minority

3 A New University, synonymous with Post-1992 University or modern university, is a former polytechnic or central institution in the United Kingdom that was given university status through the Further and Higher Education Act 1992 or an institution that has been granted university status since 1992 without receiving a royal charter (Armstrong 2008). 
men and women in comparison to white people? (5) How can the current mental health/wellbeing services provided be improved for BME individuals within universities and society more generally?

Thematic analysis was implemented to identify key themes that commonly emerged among the participants regarding perceived barriers for accessing mental health services among ethnic minorities at universities (Braun and Clarke 2014). The researcher became familiarized with the scripts and notes and developed an iterative coding scheme using NVivo qualitative data analysis software. This process involved the identification of common words and phrases expressed among the participants, which were coded and subsequently grouped into themes and sub-themes to illuminate commonalities in experiences. Upon identifying key themes, the transcripts were coded, according to the theme. It is important to note that, during the data review process, new themes and sub-themes emerged and were adapted in an iterative process. There was some acknowledgement that, as a researcher closely associated with the topic area due to being a BME individual, some organic bias may be inherent even though all protocols were administered to ensure objectivity was maintained and any potential biases were minimized (Cohen et al. 2011). In an attempt to minimize bias, another researcher was enlisted for this phase of the data analysis process. This researcher independently read the scripts and coded and analyzed the data to enhance the validity of the emerging themes and claims. Anonymized quotes from the participants were used to illustrate pertinent themes identified within the study.

\section{Mental Health Support Available at Universities}

In attempting to unpack the paucity of mental health interventions available to ethnic minorities within university spaces, which is the premise for this particular study, it is important to acknowledge the services that are generally available for all students within the higher education sector. Generally, universities will be able to provide access and signpost university students to the following external services through consultations with occupational health: counseling, psychological therapies such as cognitive behavioral therapy, and access to mental health support charities such as Young Minds, The Samaritans, and MIND (MIND 2013; Wallace et al. 2016).

\subsection{Exploring Mental Health for Black and Ethnic Minorities (BME) within Higher Education}

The study identified two broad inter-related themes as barriers for accessing mental health services adapted from the work of Memon et al. (2016) within universities: (1) personal and environmental factors and (2) relationship between the service user and healthcare provider situated around power and hierarchy. The themes identified were comprised into sub-themes, which aim to illuminate some of the perceived problems for ethnic minorities attempting to access psychological support.

An important distinction to be made through the proliferation of these findings is that there was inevitably a natural crossover between university mental health provision and NHS mental provision since many of the participants experienced similar discriminatory issues during the presentation of mental health difficulties with healthcare professionals. The nature of the research method used was not intended to be invasive but rather glean potential issues and problems for ethnic minority students when engaging with healthcare professionals regarding mental health within universities.

There was an intention initially for participants to disclose more candid accounts regarding personal experiences with mental illness. However, this did this not transpire. The researcher was advised by mental health professionals that the detailing and explaining of mental health episodes can trigger phases of trauma and destabilize psychological rehabilitation. It was advised that the recollection and reliving of periods associated with psychological distress may interfere with behavioral strategies implemented and aimed at focusing on and prioritizing the present context. It was also acknowledged by the researcher and healthcare professionals that they did not have the expertise to deal with the disclosure of particular traumatic experiences associated with mental health and psychological well-being. 


\subsubsection{Personal and Environmental Factors}

Several personal and environmental factors were recognized as affecting access to mental health services for ethnic minorities within universities. Four sub-themes were identified as potential barriers. Each is described below.

\subsubsection{Recognition of Mental Health Problems and Fear of Stigmatization}

Inability to recognize symptoms of mental illness and the subsequent denial to accept a diagnosis of mental illness in the first instance before commencing university were mentioned to be key factors in seeking help from health services. This was particularly pertinent for individuals who were fearful of stigmatization within their communities. For instance, one woman stated that:

'Negotiating mental illness is hard because, within black communities, you can become tarnished as being unstable so you are isolated ... then you arrive at university as a minority and then you become further isolated because no one looks like you and the health professionals are all white and have no experience or knowledge of your racialized plight among other things' (Female, Black, UG1).

This was compounded by a cultural belief that mental health was sometimes not to be discussed openly with family members since there could be reputational damage, which could impact 'standing' and 'status' within communities. Consequently, seeking mental health support or intervention for psychological problems was reported to be delayed, which often leads to the presentation as a crisis or emergency because people:

'Are reluctant to disclose this stuff until they reach a crisis point or rock bottom and, as a black male, you are already stigmatized and you are aware that you will be further [stigmatized] upon being diagnosed with this illness, which will consequently affect your university studies' (Male, Mixed-Heritage, UG5).

\subsubsection{Social Networks}

Social networks were considered to be an anchor for BME individuals who often felt isolated within university spaces. Individuals expressed that healthcare professionals within universities were:

'Quick to make misinformed judgements such as are you from a one-parent family ... are there gangs where you live, I know white students do not get asked this trivial stuff' (Male, Latin-American, UG 9).

Establishing networks of ethnic minority friends from similar cultural backgrounds within universities was considered to foster:

'A sense of community in what was considered to be an overwhelmingly white environment' (Female, Asian, UG, 17).

Importantly, this was considered an integral factor towards providing a safe framework for the discussion of problems. It was felt that:

'This could play a valuable role in accessing mental health services for BME individuals at university' (Female, Mixed-Heritage, PG, 2).

However, there was a perception that social networks could also be perceived as a barrier either through their absence, by demonizing health services, or by advocating alternative treatments to professional services. One participant highlighted that:

'Feelings of isolation in universities can significantly affect your judgement as you become fixated on trying to survive with friends indicating that seeking help as a person of color demonstrates a weakness that white people may exploit, which can affect your studies or attainment' (Female, Black, PG, 6). 
There was a perception that, in navigating the racialized terrain of university life within the UK as an ethnic minority student:

'The mental health services provided at universities did not always understand all of the racialized nuances involved' (Male, Mixed-Heritage, PG, 1). Further, one participant added 'that we are really, really isolated in this space as black people' (Female, Latin-American, UG, 7).

Social isolation external from university environments was also relevant for participants who lived alone or whose family structure was fractured for varying reasons.

'Personally speaking, the family structure at home is non-existent so trying to negotiate these episodes while at university is difficult as I do not really get any support or solace at home. As a result, this is affecting my grade attainment ... finding help can be so hard' (Female, Black, UG, 10).

Informal social support structures have, for many of the participants, been a reliable and viable alternative for seeking professional mental health services. This was particularly prevalent where there were historical and generational feelings of distrust within communities towards formal healthcare provision in society.

'Within African and West Indian communities ... people do not trust psychiatrists in particular. They believe in the power of prayer as the only viable intervention and this is often mapped against a relative talking about experiences of bad mental healthcare or diagnosis, re-emphasizing their need to solely place their faith or trust in religious intervention' (Female, Black, UG, 13).

\subsubsection{Gender Differences}

Many participants suggested that there was a reluctance among BME men to discuss or disclose issues aligned to mental health within university and society. Participants indicated that this reluctance was heightened when referrals to discuss issues with mental health professionals was suggested:

'Men kind of prefer keeping things to ourselves ... I always think that, as a black man, I am judged differently and I do not want to provide anyone with the ammunition to judge me differently ... even more than I am already judged' (Male, Mixed-Heritage, PG, 2).

Participants also perceived that services were not:

'Available for men of color who have mental health issues particularly at universities like ours (Russell Group) where there are not a lot of black men' (Male, Black, UG, 12).

Although a large number of male participants emphasized the importance of discussing concerns, the ability to deal with problems was often couched within the discourse associated with masculine identity. For example:

'Pride, strength, and resilience' (Male, Latin-American, UG, 5).

\subsubsection{Cultural Identity and Stigma}

Significant emphasis was placed on cultural identity and stigma towards mental health as barriers for accessing healthcare services at university. Several participants commented on how one's cultural background and identity defined acceptable responses to mental health problems and appropriate coping mechanisms. One participant indicated that:

'Within African families, there is an expectation to continuously be resilient as you are reminded of the sacrifices made for you to attend university' (Female, Black, PG, 1). 
For participants, discussions concerning mental health were often perceived as a socially unacceptable topic for discussion:

'If I ever told my parents that I was struggling to get mental health support at university, they would possibly disown me. This is not the kind of thing we openly discuss in my culture particularly as a black, female Muslim' (Female, Black, UG, 11).

Mental illness was typically viewed negatively in the participants' experiences within university and among their communities. The stigma often accompanying mental illness within communities meant that individuals were reluctant to openly acknowledge symptoms.

'For me ... the feeling of no one to turn to is difficult, none at university, none at home ... these thoughts then follow you everywhere, making the mental illness worse ... then you tell yourself I do not have a problem. You just become more stressed and isolated' (Male, Black, PG, 1).

There was a perception that the consequences of cultural stigma attached to mental health were considered as far-reaching and consequential beyond the individual. One participant explained that the residual effects of a mental health diagnosis could stigmatize an entire family, which affects networks and social capital within communities.

'In my culture, if you hear that someone within your community/family has mental illness ... you place all sorts of unfair and judgmental labels on that individual and their family' (Female, Asian, PG, 18).

\subsection{Relationship between Service User and Healthcare Provider Situated around Power and Hierarchy}

Several components framing the relationship between service user and healthcare provider by considering aspects of power and hierarchy were recognized as affecting access to mental health services. Three sub-themes were identified as potential barriers. Each is described below.

\subsubsection{Language}

Language was seen as a prominent factor with regard to accessing mental health services. Participants emphasized that, in many cases, individuals from the BME community sometimes had difficulty conversing or understanding English especially if this was adopted as a second language. The inability to effectively communicate with healthcare professionals proved to be an obstacle in being able to accurately articulate problems.

'As a student who is a recent immigrant, my understanding of English is improving daily. As a result, sometimes I struggle to articulate clearly and accurately what I would like to say to the healthcare professionals provided at university. For this reason, I prefer not to discuss my psychological issues as they will misjudge what I am saying and then heavily medicate me' (Female, Asian, UG, 4).

Consequently, individuals experiencing this will endure additional stress, which may result in withdrawing from healthcare services altogether.

'My fear is receiving an inappropriate investigation into my psychological state, which could affect my studies and potential treatments that are not cognitive or behavioral. As a result, between university and my General Practitioner (GP) ... I prefer not to say anything' (Female, Asian, UG, 4).

Among the 32 participants, they collectively noted at their institutions that there were no visible interpretation services available with regards to mental health support. Concerns were raised by one participant who mentioned: 
'This amounts to universities not recognizing their responsibility to serve a multi-cultural and diverse student population ... the inadequate provision of interpreters only highlights that universities do not value or respect the needs of ethnic minority students' (Female, Mixed-Heritage, PG, 20).

\subsubsection{Communication: Cultural Naivety, Insensitivity, and Discrimination}

Participants were explicit in describing how healthcare providers often struggled to understand BME people's experiences. Several participants verbalized their frustration in having to continuously explain:

'The Black experience ... always having to navigate racism and remain on the outside as a minority in university and society. It is exhausting and mentally grinds you down' (Female, Black, UG, 8).

Often participants felt that this lack of understanding by healthcare professionals interfered with effective therapy. They also felt poor diversification of healthcare professionals was a direct consequence of this. One participant suggested that:

'To be honest, it would just be nice to speak to a person that looked like you ... who can relate to you culturally and understand what you are experiencing as a person of color' (Male, Mixed-Heritage, UG, 9).

Typically, participants also commented on the fragility and discomfort often encountered when discussing issues of racism with healthcare professionals.

'I have also found that when you, for instance, discuss how racism impacts your daily existence ... that actually a lot of them get uncomfortable ... ' (Female, Mixed-Heritage, $\mathrm{UG}, 32)$.

Participants perceived that, within universities and the healthcare profession, generally specialists were unable to conceptualize or empathize with the realities and lived experiences of those from a BME background. Services were considered too insensitive to the needs of BME service users with a somewhat dismissive approach towards treating ethnic minorities.

'The reality is that we receive inadequate treatment because of our skin color and that is the sad reality. This just pertains to the historical deficit model concerning black people ... ' (Female, Mixed-Heritage, PG, 30).

Participants drew comparisons with white counterparts experiencing similar problems within a university.

'I have a friend studying the same degree as me and she confided in me and said she also suffers from depression and anxiety. The university doctor referred her to a specialist behavioral consultant to work with her ... I was recommended medication for the same problem ... how is that fair ... ?' (Female, Asian, UG, 18).

\subsubsection{Power and Authority}

Among the participants, power and hierarchy were considered as oppressive instruments for maintaining inequality and inequity at the expense of ethnic minorities. Interactions with clinicians were recalled as:

'Experiencing patronizing and condescending behavior from healthcare professionals' (Male, Asian, UG, 2).

There was concern that, in some cases, clinicians could potentially abuse their power. 
'Let's face it ... with the stroke of a pen you could be sectioned or sent away for some counseling. Or ... have administered some ridiculous dose of whatever kind of medicine' (Male, Black, UG, 13).

Participants expressed a need for imbalances of power and hierarchy to be challenged with greater diversification in healthcare services at universities and society.

'In addressing issues of power, particularly with ethnic minorities, I think one answer is greater diversification and the introduction of better interpretive services' (Female, Latin-American, PG, 5).

Furthermore, participants also described a sense of helplessness and passivity with regard to healthcare interactions and treatment.

'You are always in a situation where you cannot challenge what is said. Otherwise, you run the risk of being labeled difficult or unstable. In those situations, you really recognize your lack of autonomy and power' (Female, Black, PG, 29).

Challenging power dynamics was considered as a potentially worsening service user and healthcare provider relationships with exclusion from services seen as a possible consequence of assertive behavior.

'I challenged the opinion of an assessment made by a psychiatric nurse at university and then I received a letter two weeks later stating that they were no longer able to support me. At that point, I wish I had not said anything in the first place' (Female, Black, UG, 15).

Participants expressed a need for more.

'Positive, interpersonal dialogue between service users and healthcare providers' (Female, Black, UG, 27) where the consultation was 'a reciprocal dialogue' (Male, Asian, PG, 25).

This was considered to be an important factor in facilitating better ethnic minority engagement with mental health services at universities and wider society.

\section{Discussion}

The findings presented in this paper highlight how access to healthcare and psychological well-being for BME individuals inside and outside university was a function determined by personal and environmental factors and the relationship between the service user and the healthcare provider. The study not only identified key barriers for accessing mental health services that were specific to people from the BME backgrounds but also barriers likely to be shared with other minority groups such as those with a disability or marginalized populations that have historically struggled to gain access to psychological support interventions. A reoccurring narrative espoused within this study highlighted an inability by mental health professionals to recognize and accept symptoms as mental illness when engaging and diagnosing BME patients. This was perceived as a barrier to seeking help particularly for individuals who came from cultures and communities where they had felt mental illness was trivialized and stigmatized. Previous research (Bhui et al. 2003; Grey et al. 2013; Memon et al. 2016) has demonstrated that culturally specific constructs of psychological distress can determine how individuals perceive and disclose their symptoms to mental health professionals. The nature of help seeking behavior and engagement with services was an issue that was deemed to be problematic because ethnic minorities felt that they continually remained on the periphery of these services through varying environmental and societal factors.

Cultural pressures and perceptions were significant factors that affected access to mental health services. There was a perception that some cultures did not recognize symptoms associated with stress, depression, or mental illness generally. Instead, it was perceived that often constrained and 
narrow views of mental illness were normally confined to or associated with culturally unacceptable behaviors such as aggression (Keating 2007; Palmer and Ward 2007). Consequently, this meant that symptoms normally associated with mental illness remained unrecognized among families, communities, and friends. There was a perception that other interventions were preferred in favor of psychological intervention such as prayer and family and community mediations (Grey et al. 2013).

Participants did illuminate that the continuous traversing of external and environmental factors often situated within a discriminatory or racial backdrop was a significant determining factor in the onset of potential mental illness (Sivandan 1991). There was a general consensus that this became exacerbated particularly when being in a university space as an ethnic minority where it was felt that negotiating this space significantly contributes to the development of feelings associated with anxiety and stress. This aligns with the Memon et al. (2016) suggestion that mental health symptoms can be perceived and presented as somatic rather than psychiatric in origin. Individuals may find it easier to understand and express mental distress in the context of physical symptoms, which can potentially lead to a misdiagnosis. This is a common experience for BME individuals experiencing mental illness. It was perceived that this happens because of negative racialized ascriptions and stereotypes that often presented ethnic minorities as aggressive, violent, economically poorer, unemployable, and welfare dependent (Fernando 2010).

The participants perceived language as a barrier to communicating their needs to healthcare professionals and, for BME university students who have adopted English as a second language, this was particularly difficult since disclosure of mental and physical feelings were often misunderstood or misinterpreted. This has also been found in previous studies (Keating and Robertson 2004; Myrie and Gannon 2013; Yancey et al. 2006). In attempting to evaluate the spiral of discrimination encountered by BME individuals when disclosing episodes of mental illness, language becomes pivotal in accurately assessing and diagnosing mental illness. Research (Alvarez et al. 2006, Benoit et al. 2005, Bhui et al. 2004, and Williams 2005) has found that, where ethnic differences played a role in the overall experience of adult acute mental healthcare, it was largely due to language.

Within this study, participants expressed difficulties in being able to articulate symptoms and concerns with some suggesting that, on many occasions, there were overt examples of racism often involving health professionals within universities misinterpreting words said. Often, it was suggested that dialect and accents were considered to be a discriminatory reason for not wanting to understand or objectively assess symptoms before leaning to the safe ground of 'poor English' being a reason that conclusive medical judgements could not be made. Consequently, this may further prolong experiences with mental illness for BME individuals. Subsequently, this acted as a barrier in a number of ways, which induced the following: increased stress, reluctance to engage with services, and inability to access appropriate care. Expression of mental health problems is often layered in culturally specific nuances and subtlety, which can be lost within the service user and healthcare provider interaction with medical terminology sometimes providing further obstacles (Memon et al. 2016; Suresh and Bhui 2006).

The impact of cultural inference was extremely pertinent to the participants especially those whose cultures were intertwined with faith and cultural expectations. Memon et al. (2016) explains that cultural frameworks can have a significant impact on how BME individuals attempt to access help within their community and this was particularly evident within the study where participants continuously reiterated stigma as a barrier to seeking and accessing mental health services. MIND (2013) state that stigma is well recognized as a factor in determining health seeking behavior and service usage.

Importantly, the participants in this study indicated that, within ethnic minority communities, mental health can often be perceived as a socially unacceptable topic for discussion within their community. Previous studies (Bhui and Sashidharan 2003; Sainsbury Center for Mental Health 2002; Weich et al. 2004) have shown that mental health problems are often associated with negative or traumatic experiences, which can provoke significant emotional and behavioral responses from individuals and the community, which leads to discrimination and disadvantages (Braun and Clarke 2006; Wilkinson and Pickett 2010). As a consequence, Memon et al. (2016) suggest that individuals from 
ethnic minorities may conceal symptoms, delay seeking help, or fail to adhere to treatment, which can potentially lead to a delayed presentation or a crisis situation. Creating greater cultural awareness requires a dismantling of dominant discourses concerning ethnic minorities within society regarding their association with mental health services (as often many ethnic minority individuals enter the healthcare service via the judicial or criminal system). This was a point emphasized by the participants who felt that many of their experiences engaging with the health services were couched within racially discriminatory occurrences (Grey et al. 2013). Cultural naivety and insensitivity within healthcare services was recognized as a barrier for accessing services. Participants articulated that mental health services were often unresponsive to the specific and unique needs of ethnic minority groups. This aligns with studies (Arthur et al. 2010; Suresh and Bhui 2006) suggesting that individuals can perceive services to replicate discriminatory experiences frequently encountered within wider society.

In many ways, the mental health service does not, unfortunately, recognize some of the deeply-entrenched institutional racism that permeates wider society. Therefore, it is perhaps unsurprising that BME service users may perceive mental health services to be discriminatory and as representing a source of formal authority and social control (Arthur et al. 2010). Participants felt that, within universities, this was further exacerbated particularly in institutions where the majority student population was white. In such cases, individuals were more likely to avoid contact with formal services. Yet, there is research that infers that there is little evidence to suggest that service users' perceived ethnicity has been a major influence on ethnic minorities' experience of acute care (Weich et al. 2012). Conversely, other studies (Arthur et al. 2010, Cross and Bloomer 2010, and Knifton et al. 2010) have shown that service users can be acutely aware of the centrality of whiteness and how it transpires within institutions. There were similarities in the narrative posited around the university being a dominant white space and the perception that mental health services often reinforce a white Eurocentric model of healthcare, which marginalizes and provides little consideration or understanding of ethnicity (MIND 2013).

The prominent role of social networks and aspects of integration was considered pertinent. Factors associated with belonging in university and community were identified throughout the study. This was consistent with previous research (Burnett and Peel 2001, and Weich et al. 2012) especially the preference of family and friends as a source of help for common mental health problems rather than healthcare professionals. It is important to note that this preference did also coincide with judgements made by family and friends, which further increased feelings of embarrassment regarding the disclosure of mental illness. Despite these perceived judgements, participants still recognized social networks as a source of support, which facilitated the discussion of problems while endorsing access to healthcare services. Research (Cross and Bloomer 2010, and Memon et al. 2016) indicates that BME individuals often find greater commonality in discussing mental health issues within their own social networks rather with professionals despite some of the cultural reservations and stigmatizations associated with mental illness. In many cases, individuals from ethnic minorities will consider formal mental health services as a last resort (Vernon 2011).

An aspect explored throughout the study was the impact of social networks within the community and the university spaces. The possibility of social networks acting as a barrier or alternative to professional mental health services is supported by qualitative studies (Evans-Lacko et al. 2014; Memon et al. 2016; Lamb et al. 2012), which suggests that individuals from a BME background are less likely to contact their GP about mental health issues. In part, this may be due to feelings of marginalization and exclusion within universities especially those situated within majority white populations. A lack of trust in mental health services subsequently encourages BME people to sometimes utilize their friends and family first as the first point of contact.

Within this study and mental health commentaries, generally there are patterns to suggest that ethnic minority men, in particular, were a hard-to-reach group and, due to some of the societal stigmatizations which already accompany men of color, they were more reluctant to seek help with a feeling that mental services tended to exclude and stereotype them as a group. Previous studies 
(Dowrick et al. 2009; National Mental Health Development Unit 2011) suggest that men were less likely than women to seek help for common mental health problems and more likely to be resistant towards help seeking behavior. Men from ethnic minority communities ascribed particular importance to self-reliance in managing mental health issues (Dowrick et al. 2009). Perhaps, unsurprisingly, this was a perspective that was supported by the findings in this study.

Within universities, participants' felt that the relationship between service user, pastoral services, and healthcare providers was recognized as pivotal to overall experience and attainment with regards to accessing communication services. The emphasis placed on these services having greater diversification was perceived as an important factor in being able to respond effectively to ethnic minority service user needs. To this end, diversification for ethnic minorities is a significant catalyst towards effective communication, which becomes central to the service user and healthcare provider relationship. The fostering of multi-cultural and positive relationships with healthcare professionals is likely to encourage disclosure with better healthcare outcomes in terms of diagnosis and treatment (Dowrick et al. 2009). It is well known that poor communication and interaction particularly in the case of ethnic minorities' experiences as service users with health service providers becomes pivotal in encouraging or dissuading future health seeking behaviors (Dowrick et al. 2009). Fear, stereotyping, and stigmatizations within communities, social circles, and university spaces, in particular, were highlighted as a cause of poor communication with healthcare professionals. Furthermore, there was a perception that healthcare professionals were often perceived to have significant power and authority over BME service users and this was attributed to the overarching centrality and hegemony of whiteness within universities and society in general (Grey et al. 2013). The findings in this study coincide with previous research (Ahmed 2012; McIntosh 1990 and Memon et al. 2016), which indicates that this power could represent a hierarchy and present difficulty if service users or families challenged a professional diagnosis.

In this study, participants perceived mental health services within universities and society generally to be limited with regard to accommodating their diverse needs as BME service users. In particular, they felt there was always a reluctance by healthcare professionals to offer cognitive alternatives instead opting and preferring to offer drug-based treatments. Previous studies (Cooper et al. 2013; MIND 2013) provide a somewhat mixed picture. Some studies have found that BME service users were no more likely to take medication for mental health illness with a particular emphasis on potential, sedative side-effects. Conversely, others (Dowrick et al. 2009; Evans-Lacko et al. 2014; Memon et al. 2016) suggest that BME service users are more likely to receive medication and less likely to receive psychotherapy and other alternative therapies that have less sedative and personality-altering effects. Participants in this study also felt that, within universities, respective communities, and the healthcare sector, there was a limited awareness regarding the range of services available to individuals, which, consequently, limits opportunities to develop bespoke, individual care plans.

Previous studies (Evans-Lacko et al. 2014; MIND 2013, and Weich et al. 2012) also found that the availability of information about services affected accessibility of mental health services as did other geographical and physical factors such as distance. This very much echoes some of the themes illuminated within this study regarding access to services for ethnic minorities attempting to navigate university life. The UK BME population represents a highly diverse entity and the participants within this study represent a sub-section of the general population. Additionally, the majority of BME participants within this study were currently studying at university with a few having recently graduated within the last 14 months. Therefore, it is important to note that the perspectives expressed throughout may not represent the views/perceptions of all strata of the BME population.

Some previous studies (Dowrick et al. 2009; MIND 2013; Rugkåsa and Canvin 2011) have identified differences among specific ethnic minority group(s) with regard to perceptions of mental illness and stigma. This study was focused on BME individuals with a specific emphasis on those undertaking university study in undergraduate or postgraduate capacities. The objective situated within a higher 
education context was to understand the barriers and community perspectives that could be possibly applicable to the majority of the ethnic minority population within the UK.

Furthermore, we were unable to conduct a comprehensive analysis of ethnicity-specific barriers for accessing services since some of our participants for personal reasons were reluctant to respond to interview questions specifically focused on measuring the impact of culture and ethnicity regarding the retrieval of mental health support. Conversely, there were explorations regarding differences in perception of barriers to accessing mental health services by gender particularly for black men. It is important to note that, in most qualitative studies in similar settings, it is pertinent to acknowledge that results gleaned could potentially be affected by selection bias. For example, if the study included BME participants who were particularly keen to discuss their views and perceptions and/or BME participants who have had a negative experience of accessing/using mental health services and, therefore, wanted to share these experiences. Considering the aim of the study, the inclusion of a specific under-represented sample of participants and the narratives gleaned, there is scope to suggest that BME individuals are subject to significant barriers, which are situated within an institutionally racist society. Upon navigating these societal structures, ethnic minorities encounter further barriers as they become more socially mobile most notably at university where they are often isolated and within employment where they experience inequity in attempting to access mental health services. Subsequently, this points to BME individuals consistently being on the periphery of healthcare services, which means that often ethnic minorities will attempt to traverse mental illness alone.

The findings presented hope to inform the development, commissioning, and delivery of effective and culturally applicable mental health services while additionally improving access to these services for BME individuals. The study not only identified key barriers that are relevant to the BME population, but also barriers likely to be shared with other minority or marginalized groups within universities and society in general. This study was situated against a higher education backdrop, which focused on BME students as a unified group. The large majority of recent studies have been based on a particular stratum of the BME population, focusing on either a specific issue or service-user sub-group.

In contrast, this study attempted to provide a comprehensive, practical, and systematic discussion on the subject of mental health concerning BME individuals within higher education while focusing on better outcomes for the BME population as a whole regarding access to mental health services. The narratives and perspectives expressed reflect perceptions of barriers for accessing mental health services that currently point towards disrupting inequitable structures, which omit ethnic minorities from suitable psychological support. Dismantling this legacy of inequality is difficult but the findings presented advocate and endorse penetrative and policy-driven actions for improving equity in healthcare and reducing health inequalities. The findings presented also contribute to the on-going dialogue in the Department of Health and Public Health England to develop more effective interventions, which aim to promote improved mental well-being among the BME populations while addressing racial/ethnic inequality throughout the mental health system. The findings disseminated within this study could potentially provide a stimulus that assists toward informing the development of such efforts and the mental health strategy within the $\mathrm{UK}^{4}$.

4 A pertinent aspect that was not explored throughout this study was the use of Complementary and Alternative Medicine (CAM). The use of this as a mental health intervention has gained momentum and popularity globally within the healthcare profession particularly as an effective intervention for ethnic minority groups. While this particular theme was not explored due to the extensive focus on BME individuals experiencing mental health issues within university contexts, there is scope for future research pathways considering ethnic minority psychological well-being to explore the effectiveness of this intervention. The incorporation of this into future related research could examine why this particular phenomena has proven to be particularly successful among ethnic minorities. Additionally, factors concerning accessibility for ethnic minority service users for this psychological intervention could also be considered. 


\section{Conclusions and Recommendations}

Gleaning the perspectives of BME service users on barriers for accessing mental health services have the potential to translate into tangible and effective actions that can promote greater equity within the healthcare profession. It is important to focus on tackling barriers at the personal and environmental level particularly at our universities where ethnic minorities continue to experience a plethora of barriers. From a societal perspective, there must be a collective endeavor that focuses on removing barriers encountered at the interface between service users and healthcare providers (Vernon 2011). Tangible actions must be reflected through the following: raising awareness of mental health issues while reducing stigma, raising awareness of services and access pathways, diversifying healthcare staff within the mental health services, and developing cultural cognizance and sensitivity (Grey et al. 2013). BME service users need to be better informed upon entering university to become enabled and empowered to engage with their own mental health and well-being and familiarize themselves with the provided services. Raising awareness of mental health can reduce stigma and help individuals recognize symptoms. This may encourage health seeking behavior at an earlier stage of presentation (Dowrick et al. 2009).

Changing the cultural paradigm requires delivering this work at a local level and raising awareness of the locally available mental health services and the variety of pathways for accessing care. It is imperative to educate and empower individuals and communities regarding mental health support. There is a responsibility among mental health services, local authorities, healthcare services, and community groups to develop ways to collate and disseminate such information in addition to ensuring language accessibility. Additionally, financial resources must be provided for healthcare providers to undertake compulsory continuing professional development training for further understanding cultural issues and differences and the sensitive and diverse needs of ethnic minority service users.

Participants overwhelmingly within this study expressed a desire to see greater diversification regarding service providers. The participants anticipate that BME practitioners may be more empathetic towards understanding the plights and experiences of ethnic minorities. Research (Grey et al. 2013; MIND 2013) on ethnic concordance between service users and providers has found that people with a BME background who have experienced racial discrimination in healthcare have a preference for ethnic similarity regarding healthcare providers. Improved diversification would facilitate better ethnic representation in the healthcare service, which may support linguistic factors while potentially facilitating more accurate clinical judgments. Furthermore, strategies aimed at expanding and enhancing the role of BME staff within mental health services at universities in particular may help deliver more culturally sensitive interactions and services. The acceleration of improved mental health outcomes for ethnic minorities requires building trust between individuals, communities, and mental health services. This is a particularly important factor that has be accelerated with government policy (MIND 2013).

Government interventions must ensure that gaps are identified within the mental health service provision which highlight potential new and culturally appropriate interventions for ethnic minorities experiencing mental illness. Importantly, policy-makers must consider how mental health support systems can diversify. Within a university and societal context, we also need to consider how these systems can function to work against the racism and isolation in wider society that places ethnic minorities at risk in the first instance by considering the communities that are more likely to struggle and less likely to receive the support required (BME Network 2016).

Black students, and, more widely, BME people, experience mental health differently. These experiences are often situated and tinged within racist connotations. For ethnic minorities, mental health problems are deeply rooted in different systemic issues. The racial ascriptions, which stereotypically accompany BME individuals, ensure that this community is diagnosed and treated differently within mental health services with consistent experiences of poorer satisfaction and less productive outcomes (Vernon 2011). Moving forward, systemic changes are required to dismantle the landscape of institutional racism, which ensures 
that ethnic minorities continue to experience a significant disadvantage when attempting to access psychiatric services they are entitled to receive.

Funding: This research received no external funding.

Conflicts of Interest: The author declares no conflict of interest.

\section{References}

Ahmed, Sara. 2012. On Being Included: Racism and Diversity in Institutional Life. Durham and London: Duke University Press.

Alexander, Claire E. 2017. Breaking Black: The death of ethnic and racial studies in Britain. Ethnic and Racial Studies 41: 1034-54. [CrossRef]

Alexander, Claire E., and Jason Arday. 2015. Aiming Higher Race, Inequality and Diversity in the Academy. London: AHRC (Runnymede Trust, Runnymede Perspectives).

Alvarez, Roger A., Elias Vasquez, Carla C. Mayorga, Daniel J. Feaster, and Victoria B. Mitrani. 2006. Increasing minority research participation through community organization outreach. Western Journal of Nursing Research 28: 541-60. [CrossRef] [PubMed]

Andrews, Kehinde. 2016. Black Studies University Course Long Overdue. Available online: https: //www.theguardian.com/commentisfree/2016/may/20/black-studies-university-course-long-overdue (accessed on 17 December 2017).

Arday, Jason, and Heidi Safia Mirza, eds. 2018. Dismantling Race in Higher Education: Racism, Whiteness and Decolonising the Academy. London: Palgrave Macmillan.

Arday, Jason. 2017. University and College Union (UCU): Exploring Black and Minority Ethnic (BME) Doctoral Students' Perceptions of a Career in Academia: Experiences, Perceptions and Career Progression. London: Creative Commons.

Armstrong, Catherine. 2008. What Is a University in the UK? Available online: https://web.archive.org/ web/20100513000141/http://www.jobs.ac.uk/careers/articles/1135/what-is-a-university-in-the-uk/ (accessed on 12 January 2018).

Arthur, Carlotta M., Frederick W. Hickling, Hilary Robertson-Hickling, Tammy Haynes-Robinson, Wendel Abel, and Rob Whitley. 2010. 'Mad, sick, head nuh good': Mental illness stigma in Jamaican communities. Transcultural Psychiatry 47: 252-75. [CrossRef] [PubMed]

Benoit, Cecilia, Mikael Jansson, Alison Millar, and Rachel Phillips. 2005. Community-academic research on hard to reach populations: Benefits and challenges. Qualitative Health Research 15: 263-82. [CrossRef] [PubMed]

Bhopal, K. 2014. The Experience of BME Academics in Higher Education: Aspirations in the Face of Inequality. Stimulus paper. London: Leadership Foundation for Higher Education.

Bhui, Kamaldeep, and Sashi P. Sashidharan. 2003. Should there be separate psychiatric services for ethnic minority groups? British Journal of Psychiatry 182: 10-12. [CrossRef] [PubMed]

Bhui, Kamaldeep, Kwame McKenzie, and Paramjit Gill. 2004. Delivering mental health services for a diverse society. British Medical Journal 329: 363-64. [CrossRef] [PubMed]

Bhui, Kamaldeep, Stephen Stansfeld, Sally Hull, Stefan Priebe, Funke Mole, and Gene Feder. 2003. Ethnic variations in pathways to and use of specialist mental health services in the UK. Systematic review. British Journal of Psychiatry 182: 105-16. [CrossRef] [PubMed]

BME Network. 2016. NHS BME Network. Available online: http:/ /www.nhsbmenetwork.org.uk (accessed on 7 May 2018).

Bradby, Hannah. 2003. Describing ethnicity in health research. Ethnicity and Health 8: 5-13. [CrossRef] [PubMed]

Braun, Virginia, and Victoria Clarke. 2006. Using thematic analysis in psychology. Qualitative Research in Psychology 3: 77-101. [CrossRef]

Braun, Virginia, and Victoria Clarke. 2014. What can 'thematic analysis' offer health and wellbeing researchers? International Journal of Qualitative Studies on Health and Wellbeing 9: 121-32. [CrossRef] [PubMed]

Burnett, Angela, and Michael Peel. 2001. Health needs of asylum seekers and refugees. British Medical Journal 322: 544-57. [CrossRef] [PubMed]

Care Quality Commission. 2011. Count Me In 2010 Census. Available online: http://www.cqc.org.uk/ organisations-we-regulate/mental-health-services/count-me-2010-census (accessed on 23 January 2018).

Cohen, Louis, Lawrence Manion, and Keith Morrison. 2011. Research Methods in Education, 7th ed. Abingdon: Routledge. 
Cooper, Claudia, Nicola Spiers, Gill Livingston, Rachel Jenkins, Howard Meltzer, Terry Brugha, Sally McManus, Scott Weich, and Paul Bebbington. 2013. Ethnic inequalities in the use of health services for common mental disorders in England. Social Psychiatry and Psychiatric Epidemiology 48: 685-92. [CrossRef] [PubMed]

Cross, Wendy M., and Melissa J. Bloomer. 2010. Extending boundaries: Clinical communication with culturally and linguistically diverse mental health clients and carers. International Journal of Mental Health Nursing 19: 268-77. [CrossRef] [PubMed]

Davies, Betty, Judith Larson, Nancy Contro, Carlos Reyes-Hailey, Arthur R. Ablin, Catherine A. Chesla, Barbara Sourkes, and Harvey Cohen. 2009. Conducting a qualitative culture study of pediatric palliative care. Qualitative Health Research 19: 5-16. [CrossRef] [PubMed]

Department of Health. 2010. Delivering Race Equality Action Plan: A Five Year Review. Available online: http://www.nmhdu.org.uk/silo/files/race-equality-action-plan-a-five-year-review.pdf (accessed on 27 March 2018).

Dowrick, Christopher, Linda Gask, and Suzanne Edwards. 2009. Researching the mental health needs of hard-to-reach groups: Managing multiple sources of evidence. BMC Health Services Research 9: 226. [CrossRef] [PubMed]

Equality Challenge Unit. 2015. Equality in Higher Education: Statistical Report, Staff and Students. Available online: http:/ /www.ecu.ac.uk/publications/equality-higher-education-statistical-report-2015/ (accessed on 21 January 2018).

Evans-Lacko, Sara, Elizabeth Corker, Paul Williams, Claire Henderson, and Graham Thornicroft. 2014. Effect of the time to change anti-stigma campaign on trends in mental-illness-related public stigma among the English population in 2003-13: An analysis of survey data. Lancet Psychiatry 1: 121-28. [CrossRef]

Fernando, Suman. 2003. Cultural Diversity, Mental Health and Psychiatry: The Struggle against Racism. Hove: Brunner-Routledge.

Fernando, Suman. 2010. Mental Health, Race and Culture. London: Palgrave Macmillan.

Giuliano, Anna R., Noreen Mokuau, Claire Hughes, Guillermo Tortolero-Luna, Betsy Risendal, Reginald CS Ho, Theresa Elaine Prewitt, and Worta J. Mccaskill-Stevens. 2000. Participation of minorities in cancer research: The influence of structural, cultural and linguistic factors. Annals of Epidemiology 10: 22-34.

Glover, Gyles, and Felicity Evison. 2009. Use of New Mental Health Services by Ethnic Minorities in England. Durham: North East Public Health Observatory.

Grey, Tracy, Hári Sewell, Gillian Shapiro, and Fahmida Ashraf. 2013. Mental Health Inequalities Facing UK Minority Ethnic Populations: Causal Factors and Solutions. Journal of Psychological Issues in Organizational Culture 3: 146-57. [CrossRef]

Jones, H. Gwynne, and J. Michael Berry. 1986. Regional secure units: The emerging picture. In Current Issues in Clinical Psychology IV. Edited by Edwards G. London: Plenum Press.

Keating, Frank, and David Robertson. 2004. Fear, black people and mental illness: A vicious circle. Health and Social Care in the Community 12: 439-447. [CrossRef] [PubMed]

Keating, Frank. 2007. African and African Caribbean Men and Mental Health. Better Health Briefing Paper 5. Available online: http:/ / www.raceeequalityfoundaiton.org.uk/ (accessed on 4 February 2018).

Knifton, Lee, Mhairi Gervais, Karen Newbigging, Nuzhat Mirza, Neil Quinn, Neil Wilson, and Evette Hunkins-Hutchison. 2010. Community conversation: Addressing mental health stigma with ethnic minority communities. Social Psychiatry and Psychiatric Epidemiology 45: 497-504. [CrossRef] [PubMed]

Lamb, Jonathan, Peter Bower, Anne Rogers, Christopher Dowrick, and Linda Gask. 2012. Access to mental health in primary care: a qualitative meta-synthesis of evidence from the experience of people from 'hard to reach' groups. Health (London) 16: 76-104. [CrossRef] [PubMed]

Law, Ian. 2017. Building the Anti-racist University, action and new agendas. Race Ethnicity and Education 20: 332-43. [CrossRef]

Lent, Robert W. 2004. Toward a unifying theoretical and practical perspective on well-being and psychosocial adjustment. Journal of Counselling Psychology 51: 482-63. [CrossRef]

McIntosh, Peggy. 1990. White Privilege: Unpacking the Invisible Knapsack. In B. Schinder, An Anthology: Race in the First Person. New York: Crown Trade Paperbacks. 
McKenzie, Kwame, Chiara Samele, Elizabeth Van Horn, Theresa Tattan, Jim Van Os, and Robin Murray. 2001. Comparison of the outcome of the treatment of psychosis for people of Caribbean origin living in the UK and British Whites: Report from the UK700 trial. British Journal of Psychiatry 178: 160-65. Available online: http:/ / bjp.rcpsych.org (accessed on 29 January 2018). [CrossRef] [PubMed]

McKenzie, Kwame, and Natasha Crowcroft. 1996. Describing race, ethnicity, and culture in medical research. British Medical Journal 312: 1054. Available online: http://www.bmj.com (accessed on 4 January 18). [CrossRef] [PubMed]

Memon, Anjum, Katie Taylor, Lisa M. Mohebati, Josefin Sundin, Max Cooper, Thomas Scanlon, and Richard de Visser. 2016. Perceived barriers to accessing mental health services among Black and Minority Ethnic (BME) communities: A qualitative study in Southeast England. BMJ Open 6: e012337. [CrossRef] [PubMed]

MIND. 2012. Statistics 3: Race, Culture and Mental Health [Fact Sheet]. Available online: http://www.mind. org.uk/help/people_groups_and_communities/statistics_3_race_culture_and_mental_health (accessed on 3 January 2018).

MIND. 2013. Mental Health Crisis Care: Commissioning Excellence for Black and Minority Ethnic Groups. London: MIND.

Mirza, Heidi Safia. 2017. One in a million: A journey of a post-colonial woman of colour in the white academy. In Inside the Ivory Tower: Narratives of Women of Colour Surviving and Thriving in Academia. Edited by Deborah Gabriel and Shirley Anne Tate. London: Trentham UCL Press.

Myrie, Chanelle V., and Kenneth N. Gannon. 2013. "Should I really be here?" Exploring the relationship between Black men's conceptions of wellbeing, subject positions and help-seeking behaviour. Diversity and Equality in Health and Care 10: 1-22.

National Mental Health Development Unit. 2011. National Evaluation of the Community Development Workers. London: National Mental Health Development Unit.

Norman, Ian, and Iain Ryrie. 2009. The art and science of mental health nursing: Reconciliation of two traditions in the cause of public health. International Journal of Nursing Studies 46: 1537-40. [CrossRef] [PubMed]

Palmer, David, and Kim Ward. 2007. 'Lost': listening to the voices and mental health needs of forced migrants in London. Medicine, Conflict and Survival 23: 198-212. [CrossRef] [PubMed]

Picower, Bree. 2009. The unexamined whiteness of teaching: How white teachers maintain and enact dominant racial ideologies. Race Ethnicity and Education 12: 197-215. [CrossRef]

Pilkington, Andrew. 2013. The interacting dynamics of institutional racism in higher education. Race, Ethnicity and Education 16: 225-45. [CrossRef]

Rollock, Nicola. 2016. How Much Does Your University do for Racial Equality? Available online: https: / www.theguardian.com/higher-education-network/2016/jan/19/how-much-does-youruniversity-do-for-racial-equality/ (accessed on 10 February 2018).

Rugkåsa, Jorun, and Krysia Canvin. 2011. Researching mental health in minority ethnic communities: Reflections on recruitment. Qualitative Health Research 21: 132-43.

Sainsbury Center for Mental Health. 2002. Breaking the Circles of Fear: A Review of the Relationship between Mental Health Services and African and Caribbean Communities. London: Sainsbury Centre for Mental Health. Available online: http:/ / www.centreformentalhealth.org.uk/pdfs/breaking_the_circles_of_fear.pdf (accessed on 12 March 2018).

Sewell, Hári. 2012. Race and ethnicity in mental health care. In Working in Mental Health: Policy and Practice in a Changing Environment. Edited by Peter Phillips, Tom Sandford and Claire Johnston. Oxon: Routledge, pp. 104-15.

Shavers, Vickie L., Charles F. Lynch, and Leon F. Burmeister. 2001. Racial differences in factors that influence willingness to participate in medical research studies. Annals of Epidemiology 12: 248-56. Available online: http:/ / www.annalsofepidemiology.org (accessed on 19 April 2018). [CrossRef]

Shilliam, Robbie. 2015. Black Academia in Britain. The Disorder of Things. Available online: https: //thedisorderofthings.com/2014/07/28/black-academia-in-britain/ (accessed on 14 April 2018).

Sivandan, A. 1991. Black Struggles against Racism. Edited by Curriculum Development Project Steering Group. Setting the Context. London: CCETSW.

Suresh, Kallur, and Kamaldeep Bhui. 2006. Ethnic minority patients' access to mental health services. Psychiatry 5: 413-16. [CrossRef]

Tate, Shirley Anne, and Paul Bagguley. 2017. Building the anti-racist university: Next steps. Race Ethnicity and Education 20: 289-99. [CrossRef] 
The Russell Group. 2018. What Are We About? Available online: https://russellgroup.ac.uk/about/ (accessed on 17 February 2018).

Vernon, Patrick. 2011. Put Race Equality in Mental Health Back on the Agenda. Available online: http:/ / www. guardian.co.uk/society/joepublic/2011/mar/01/race-equality-mental-health (accessed on 23 March 2018).

Wallace, Stephanie, James Nazroo, and Laia Bécares. 2016. Cumulative effect of racial discrimination on the mental health of ethnic minorities in the United Kingdom. American Journal of Public Health 106: 1294-300. [CrossRef] [PubMed]

Weich, Scott, Laura Griffith, Martin Commander, Hannah Bradby, Sashi. P. Sashidharan, Sarah Pemberton, and Kamaldeep Singh Bhui. 2012. Experiences of acute mental health care in an ethnically diverse inner city: qualitative interview study. Social Psychiatry and Psychiatric Epidemiology 47: 119-28. [CrossRef] [PubMed]

Weich, Scott, James Nazroo, and Kerry Sproston. 2004. Common mental disorders and ethnicity in England: The EMPIRIC study. Journal of Clinical Psychology in Medical Settings 34: 1543-51. [CrossRef]

Wendler, David, Raynard Kington, Jennifer Madans, Gretchen Van Wye, Heidi Christ-Schmidt, Laura A. Pratt, Otis W. Brawley, Cary P. Gross, and Ezekiel Emanuel. 2006. Are racial and ethnic minorities less willing to participate in health research? Public Library of Science Medicine 3: 19-27. [CrossRef] [PubMed]

Wilkinson, Richard, and Kate Pickett. 2010. The Spirit Level: Why Equality Is Better for Everyone. London: Penguin Books.

Williams, Charmaine C. 2005. Ethical considerations in mental health research with racial and ethnic minority communities. Community Mental Health Journal 41: 509-20. [CrossRef] [PubMed]

Yancey, Antronette K., Alexander N. Ortega, and Shiriki K. Kumanyika. 2006. Effective recruitment and retention of minority research participants. Annual Review of Public Health 27: 1-28. [CrossRef] [PubMed]

(C) 2018 by the author. Licensee MDPI, Basel, Switzerland. This article is an open access article distributed under the terms and conditions of the Creative Commons Attribution (CC BY) license (http:/ / creativecommons.org/licenses/by/4.0/). 